\title{
NeuroImage
}

\section{Mechanisms of evoked and induced responses in MEG/EEG}

\author{
Olivier David, James M. Kilner, ${ }^{*}$ and Karl J. Friston \\ Wellcome Department of Imaging Neuroscience, Institute of Neurology, 12 Queen Square, London WC1N 3BG, UK
}

Received 14 March 2005; revised 19 October 2005; accepted 13 February 2006

Available online 24 April 2006

Cortical responses, recorded by electroencephalography and magnetoencephalography, can be characterized in the time domain, to study event-related potentials/fields, or in the time-frequency domain, to study oscillatory activity. In the literature, there is a common conception that evoked, induced, and on-going oscillations reflect different neuronal processes and mechanisms. In this work, we consider the relationship between the mechanisms generating neuronal transients and how they are expressed in terms of evoked and induced power. This relationship is addressed using a neuronally realistic model of interacting neuronal subpopulations. Neuronal transients were generated by changing neuronal input (a dynamic mechanism) or by perturbing the systems coupling parameters (a structural mechanism) to produce induced responses. By applying conventional timefrequency analyses, we show that, in contradistinction to common conceptions, induced and evoked oscillations are perhaps more related than previously reported. Specifically, structural mechanisms normally associated with induced responses can be expressed in evoked power. Conversely, dynamic mechanisms posited for evoked responses can induce responses, if there is variation in neuronal input. We conclude, it may be better to consider evoked responses as the results of mixed dynamic and structural effects. We introduce adjusted power to complement induced power. Adjusted power is unaffected by trial-totrial variations in input and can be attributed to structural perturbations without ambiguity.

(C) 2006 Elsevier Inc. All rights reserved.

\section{Introduction}

Cortical oscillatory activity, as disclosed by local field potentials (LFPs), electroencephalographic (EEG) and magnetoencephalographic (MEG) recordings, can be categorized as ongoing, evoked or induced oscillations (Galambos, 1992; Tallon-Baudry and Bertrand, 1999). Evoked and induced oscillations differ in their phase-relationships to the stimulus. Evoked oscillations are phase locked to the stimulus, whereas induced oscillations are not. Operationally, these two phenomena are revealed by the order of trial averaging and spectral analysis. To

\footnotetext{
* Corresponding author. Fax: +44 02078131445.

E-mail address: j.kilner@fil.ion.ucl.ac.uk (J.M. Kilner).

Available online on ScienceDirect (www.sciencedirect.com).
}

estimate evoked power, the MEG/EEG signal is first averaged over trials and then subject to time-frequency analysis to give an eventrelated response (ERR). To estimate induced oscillations, the time-frequency decomposition is applied to each trial and the ensuing power is averaged across trials. The power of evoked and background components are subtracted from this total power to reveal induced power. In short, evoked responses can be characterized as the power of the average; while induced responses are the average power that cannot be explained by the power of the average.

A common conception is that evoked oscillations reflect a stimulus-locked ERR, in time-frequency space and that induced oscillations are generated by some distinct high-order process. Following Singer and Gray (1995), this process is often described in terms of 'binding' and/or neuronal synchronization. The tenet of the binding hypothesis is that coherent firing patterns can induce large fluctuations in the membrane potential of neighboring neurons which, in turn, facilitate synchronous firing and information transfer (as defined operationally in Varela, 1995). Oscillatory activity that is classified as induced is the measured correlate of these massively synchronous neuronal assemblies. Oscillations are induced because their self-organized emergence is not evoked directly by the stimulus but induced vicariously through nonlinear and possibly autonomous mechanisms.

Here, we propose an alternative view that evoked and induced responses are, perhaps, more related than previously thought and that a mixture of mechanisms can generate both. Critically, we make a distinction between the mechanisms causing neuronal transients and how the response is measured operationally, in terms of evoked and induced oscillations. Having established this distinction, we then examine the relationship between the mechanisms and the time-frequency characterizations.

To pursue this, we used a model neuronal system in which the mechanisms generating responses were under experimental control. This model was a neural mass model that we have used in previous studies to look at measures of linear and nonlinear coupling in EEG/MEG (David et al., 2004). The mechanisms of phase locking in the genesis of ERPs and other phenomena (David et al., 2005). Furthermore, this model is the basis of the forward model in the dynamic causal modeling of ERP data in SPM (http:// www.fil.ion.ucl.ac.uk/spm; David et al., in press). Neuronal models play a necessary role in this context because they afford direct 
access to the processes and mechanisms producing evoked and induced oscillations. Once the mechanisms responsible for induced responses are established, one can assess the specificity of their expression in evoked and induced response components.

\section{Overview}

This paper is divided into three sections. In the first, we establish a key distinction between dynamic mechanisms, normally associated with classical evoked responses like the ERP and structural mechanisms implicit in the genesis of induced responses. Dynamic effects are simply the effect of inputs on a systems response. Conversely, structural mechanisms entail a transient change in the systems causal structure, i.e., its parameters (e.g., synaptic coupling). These changes could be mediated by nonlinear effects of input. We relate the distinction between dynamic and structural mechanisms to series of dichotomies in dynamical system theory and neurophysiology. These include the distinction between driving and modulatory effects in the brain. This section concludes with a review of how neuronal responses are characterized operationally, in terms of evoked and induced power, and how these characterizations relate to dynamic and structural perturbations.

In the second section, we show that structural mechanisms can indeed produce induced oscillations. In the example provided, responses are induced by a stimulus-locked modulation of the backward connections from one source to another. However, we show that this structural effect is also expressed in evoked oscillations when dynamic and structural effects interact. In the final section, we show the converse, namely that dynamic mechanisms can produce induced oscillations, even in the absence of structural effects. This can occur when trial-to-trial variations in input suppress high-frequency responses after averaging. Our discussion focuses on the rather complicated relationship between the two types of mechanisms that can cause responses in EEG/MEG and the ways in which evoked and induced responses are measured. We introduce adjusted power as a complement to induced power that resolves some of these ambiguities.

\section{Theory}

\section{Dynamic and structural mechanisms}

In this section, we introduce two distinct mechanisms that underlie neuronal transients. The distinction arises from a simple view of neuronal responses, as the response of an input-stateoutput system to perturbations. Any analytic system can be described by an equation governing the dynamics of its states and a function that converts the current state $x$ of the system into some output or measure $y$, in our case an EEG/MEG signal.

$\dot{x}=f(x, u, \theta)$

$y=\lambda(x, \theta)+\varepsilon$

These states $x(t)$ cover all the variables that describe the state of a neuronal system (e.g., a collection of neuronal subpopulations that constitutes an EEG source). For example, the states could include the depolarization of all the systems neuronal compartments, and any other variable that shapes its dynamics. $u(t)$ or inputs enter the state equations to changes states, directly or indirectly. This input can have both stochastic and deterministic (i.e., stimulus locked) components. $\theta(t)$ is a system parameter that encodes its functional or causal architecture; for example, the connection strengths among neuronal units. $\varepsilon(t)$ represents observation noise. See Fig. 1 for a schematic representation of these quantities.

From Eq. (1), it is immediately clear that the states, and implicitly the systems response, can only be changed by perturbing $u(t)$ or $\theta(t)$. We will refer to these as dynamic and structural effects respectively. This distinction arises in a number of different contexts. From a purely dynamical point of view, transients elicited by dynamic effects are the systems response to input changes; for example, the presentation of a stimulus in an ERP study. If the system is dissipative and has a stable fixed point, then the response is a generalized convolution of the input.

$$
\begin{aligned}
y(t)= & h(u, \theta)+\varepsilon h(u, \theta)=\sum_{i} \int_{0}^{t} \cdots \int_{0}^{t} \kappa_{i}\left(\sigma_{1}, \ldots, \sigma_{i}\right) \\
& \times u\left(t-\sigma_{1}\right), \ldots, u\left(t-\sigma_{i}\right) d \sigma_{1}, \ldots, d \sigma_{i} \kappa_{i}\left(\sigma_{1}, \ldots, \sigma_{i}, \theta\right) \\
= & \frac{\partial^{i} y(t)}{\partial u\left(t-\sigma_{1}\right), . ., \partial u\left(t-\sigma_{i}\right)}
\end{aligned}
$$

where $\kappa\left(\sigma_{1}, \ldots, \sigma_{n}, \theta\right)$ is called the $n$th order Volterra kernel. This equation may look complicated, but it is just a generalization of a conventional convolution equation to second and high-orders and obtains from a simple Taylor expansion of Eq. (1). See Friston (2001) for a fuller discussion. This generalized convolution has an equivalent representation in the frequency or spectral domain. Introducing the spectral density representation $s(\omega)$

$u(t)=\int_{s_{u}}(\omega) e^{-j \omega} d \omega$

we can rewrite the Volterra expansion, Eq. (2) as

$\begin{aligned} h(u, \theta)= & \sum_{i} \underset{-\pi}{\pi} \ldots \int_{-\pi}^{\pi} e^{j\left(\omega_{1}+, . .,+\omega_{i}\right) t} \Gamma_{1}\left(\omega_{1}, \ldots, \omega_{i}\right) \\ & \times s_{u}\left(\omega_{1}\right), \ldots, s_{u}\left(\omega_{i}\right) d \omega_{1}, \ldots, d \omega_{i}\end{aligned}$

where the functions

$\Gamma_{1}\left(\omega_{1}, \theta\right)=\int_{0}^{\infty} e^{-j \omega_{1} \sigma_{1}} \kappa_{1}\left(\sigma_{1}\right) d \sigma_{1}$

$\Gamma_{2}\left(\omega_{1}, \omega_{2}, \theta\right)=\int_{0}^{\infty} \int_{0}^{\infty} e^{-j\left(\omega_{1} \sigma_{1}+\omega_{2} \sigma_{2}\right)} \kappa_{2}\left(\sigma_{1}, \sigma_{2}\right) d \sigma_{1} d \sigma_{2}$

are the Fourier transforms of the kernels. These functions are called generalized transfer functions and mediate the expression of frequencies in the output given those in the input. Critically, the influence of high-order kernels or equivalently generalized transfer functions means that a given frequency in the input can induce a different frequency in the output. A simple example of this would be squaring a sine wave input to produce an output of twice the frequency.

The duration and form of the resulting dynamics effect depends on the dynamical stability of the system to perturbations of its states (i.e., how the systems trajectories change with the state). Structural effects depend on structural stability (i.e., how the systems trajectories change with the parameters). Systematic changes in the parameters can produce systematic changes in the response, even in the absence of input. For systems that show autonomous (i.e., periodic or chaotic) dynamics, changing the 

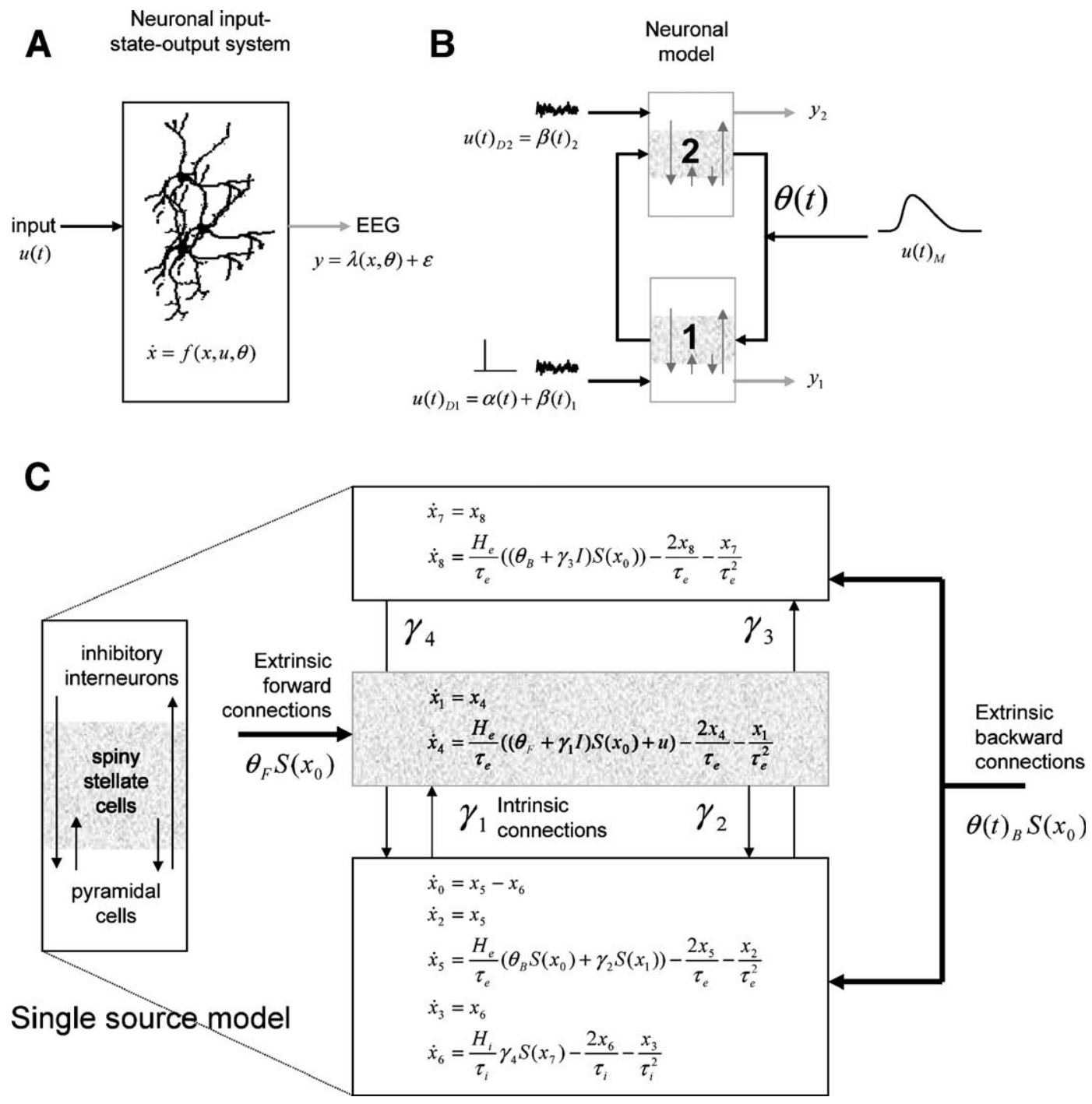

Fig. 1. (A) The causal structure of an input-state-output system is defined by parameters $\theta$. It receives inputs $u$ that can elicit changes in the states $x$ directly (e.g., driving inputs $u_{D}$, which generate postsynaptic potentials) or modulate the systems parameters (e.g., modulatory inputs $u_{M}$ which smoothly modify dynamics, through changes in synaptic efficacy). The MEG/EEG output $y$ depends on both inputs and parameters. (B) Neuronal model used in the simulations. Two cortical areas interact with forward and backward connections. Both areas receive a stochastic input $\beta$, which simulates ongoing activity from other brain areas. In addition, area 1 receives a stimulus $\alpha$, modeled as a delta function. A single modulatory effect is considered. It simulates a stimulus-related slow modulation of extrinsic backward connectivity. The outputs of the neuronal system are the MEG/EEG signals $y_{1}$ and $y_{2}$ from both areas. The model used comprises three subpopulations for each area, coupled with intrinsic connections. Approximate state equations for this model are found in David and Friston, 2003. (C) Schematic of a single source model. This schematic includes the differential equations describing the dynamics of the source or regions states. Each source is modeled with three subpopulations (pyramidal, spiny-stellate and inhibitory interneurons) as described in (Jansen and Rit, 1995). These have been assigned to granular and agranular cortical layers, which receive forward and backward connection respectively. See David and Friston (2003) for a detailed explanation of the state equations. For simplicity, we have removed lateral connections, which were not used in these simulations.

parameters is equivalent to changing the attractor manifold, which induces a change in the systems states. We before have discussed this in the context of nonlinear coupling and classical neuromodulation (Friston, 1997; Breakspear et al., 2003). For systems with fixed points and Volterra kernels, changing the parameters is equivalent to changing the kernels and transfer functions. This changes the spectral density relationships between the inputs and outputs. As such, structural effects are clearly important in the genesis of induced oscillations because they can produce frequency modulation of ongoing activity that does not entail phase locking to any event.

This difference between dynamic and structural effects is closely related to the distinction between linear and nonlinear mechanisms, but they are not synonymous. The second-order approximation of Eq. (1) makes their relationship clear ${ }^{1}$

$$
\begin{aligned}
\dot{x}= & u_{D} \partial f / \partial u_{D}+u_{D}^{2} \partial^{2} f / \partial u_{D}^{2}+\ldots \\
& +\left(J+u_{M} \partial J / \partial u_{M}+\ldots+x_{1} \partial J / \partial x_{1}+\ldots\right) x \\
J= & \partial f / \partial x
\end{aligned}
$$

Here, $J$ is the system's Jacobian. The top line encodes dynamic effects that are mediated by a dynamic input $u(t)_{D}$. This can have

\footnotetext{
${ }^{1}$ For simplicity we consider a single dynamic input $u_{D}$ and a single modulatory input $u_{M}$, which could be the same, i.e., $u_{D}=u_{M}$.
} 
both linear and nonlinear components. The second line represents structural effects that are mediated by structural inputs $u(t)_{M}$. Here, the input does not change the states directly but changes them indirectly by modulating the systems Jacobian (i.e., its dynamic structure). The matrices $\partial J(\theta) / \partial u_{M}$ could be regarded as parameters of the system or, more intuitively, as changes in the architecture induced by inputs. Critically, structural effects are always nonlinear and involve an interaction with the states. For readers familiar with dynamic causal modeling with the bilinear model, bilinear effects are structural effects. These effects are often construed as the modulation of a coupling, in a neuronal network, by an experimental input. In terms of the spectral formulation, structural inputs have only second or high-order kernels and associated transfer functions.

In summary, dynamic effects are expressed directly on the states and conform to a convolution of inputs to form responses. Structural effects are expressed indirectly, through the Jacobian, and are inherently nonlinear, inducing high-order kernels and associated transfer functions.

\section{Drivers and modulators}

The distinction between dynamic and structural inputs speaks immediately to the difference between 'drivers' from 'modulators' (Sherman and Guillery, 1998). In sensory systems, a driver ensemble can be identified as the transmitter of receptive field properties. For instance, neurons in the lateral geniculate nuclei drive primary visual area responses, in the cortex, so that retinotopic mapping is conserved. Modulatory effects are expressed as changes in certain aspects of information transfer, by the changing responsiveness of neuronal ensembles in a context-sensitive fashion. A common example is attentional gain. Other examples involve extraclassical receptive field effects that are expressed beyond the classical receptive field. Generally, these are thought to be mediated by backward and lateral connections. In terms of synaptic processes, it has been proposed that the postsynaptic effects of drivers are fast (ionotropic receptors), whereas those of modulators are slower and more enduring (e.g., metabotropic receptors). The mechanisms of action of drivers refer to classical neuronal transmission, either biochemical or electrical, and are well understood. Conversely, modulatory effects can engage a complex cascade of highly nonlinear cellular mechanisms (Turrigiano and Nelson, 2004). Modulatory effects can be understood as transient departures from homeostatic states, lasting hundreds of milliseconds, due to synaptic changes in the expression and function of receptors and intracellular messaging systems.

Classical examples of modularity mechanisms involve voltagedependent receptors, such as NMDA receptors. These receptors do not cause depolarization directly (cf., a dynamic effect) but change the units sensitivity to depolarization (i.e., a structural effect). It is interesting to note that backward connections, usually associated with modulatory influences, target supragranular layers in the cortex where NMDA receptors are expressed in greater proportion.

Having established the difference between dynamics and structural effects and their relationship to driving and modulatory afferents in the brain, we now turn to the characterization of evoked and induced responses in terms of time-frequency analyses.

\section{Evoked and induced responses}

The criterion that differentiates induced and evoked responses is the degree to which oscillatory activity is phase locked to the stimulus over trials. An ERR is the waveform that is expressed in the EEG signal after every repetition of the same stimulus. Due to physiological and measurement noise, the ERR is often only evident after averaging over trials. More formally, the evoked response $y(t)_{e}$ to a stimulus is defined as the average of measured responses in each trial $y(t)$

$y(t)_{e}=\langle y(t)\rangle$

where $t$ is peristimulus time.

A time-frequency representation $s(\omega, t)$ of a response $y(t)$ obtains by successively filtering $y(t)$ using a kernel or filter-bank parameterized by frequencies $\omega_{j}=2 \pi v_{j}$, over the frequency range of interest:

$s(\omega, t)=\left[\begin{array}{c}k\left(\omega_{1}, t\right) \otimes y(t) \\ \vdots \\ k\left(\omega_{J}, t\right) \otimes y(t)\end{array}\right]$

where $\otimes$ denotes linear convolution. $k\left(\omega_{j}, t\right)$ can take several forms (Kiebel et al., 2005). We used the Morlet wavelet:

$k\left(\omega_{j}, t\right)=\sqrt{v_{j}} \exp \left(-\frac{1}{2}\left(t v_{j} / \sigma\right)^{2}\right) \exp \left(i \omega_{j} t\right)$.

$\sigma$ is a user-specified constant, which sets the number of cycles of the wavelet, and therefore the temporal and frequency resolution of the wavelet transform. The total power, averaged over trials and the power of the average are respectively

$g(\omega, t)_{T}=\langle s(\omega, t) s(\omega, t) *\rangle$

$g(\omega, t)_{e}=\langle s(\omega, t)\rangle\langle s(\omega, t) *\rangle$

where * denotes the complex conjugate. $g(\omega, t)_{e}$ is evoked power and is simply the power of $y(t)_{e}$. Induced power $g(\omega, t)_{i}$ is defined as the component of total power that cannot be explained by baseline and evoked power ${ }^{2}$. This implicitly partitions total power into three orthogonal components (induced, baseline and evoked).

$g(\omega, t)_{i}=g(\omega, t)_{T}-g(\omega)_{b}-g(\omega, t)_{e}$

$g(\omega, t)_{T}=g(\omega, t)_{i}+g(\omega, t)_{e}+g(\omega)_{b}$

Baseline power $g(\omega)_{b}$ is a frequency-specific constant due to ongoing activity and experimental noise, both of which are assumed to be stationary, that is usually calculated over a period of time preceding stimulus presentation.

\section{Evoked and induced power and their mechanisms of generation}

In this subsection, we establish how dynamic and structural mechanisms are expressed in terms of evoked and induced power. As illustrated in Fig. 1, the inputs for the $i$ th trial $u(i)$ can be decomposed into a deterministic stimulus-related component $\alpha$ and

\footnotetext{
${ }^{2}$ A different definition is sometimes used, where induced responses are based on the difference in amplitude between single trials and the ERR: $y(t)-y(t)_{e}$ (Truccolo et al., 2002). The arguments in this work apply to both formulations. However, it is simpler for us to use Eq. (10) because it discounts ongoing activity. This allows us to develop the arguments by considering just one trial type (as opposed to differences between trial types).
} 
trial-specific background activity $\beta^{(i)}$, which is stochastic and unrelated to the stimulus

$u^{(i)}=\alpha+\beta^{(i)}$.

For simplicity, we will assume that the state-space defined by Eq. (1) operates largely in its linear regime, as suggested by studies which have found only weak nonlinearities in EEG oscillations (Breakspear and Terry, 2002; Stam et al., 1999). This allows us to focus on the first-order kernels and transfer functions. We will also assume the background activity is stationary. In this instance, the total power is, by Eq. (4)

$g(\omega, t)_{T}=|\Gamma(\omega, t)|^{2} g(\omega, t)_{u}+g(\omega)_{\varepsilon}$

$g(\omega, t)_{u}=g(\omega, t)_{\alpha}+g(\omega)_{\beta}$

In words, the total power is the power of the input, modulated by the transfer function $|\Gamma(\omega, t)|^{2}$, plus the power of the noise term. The power of the input is simply the power of the deterministic component, at time $t$, plus the power of ongoing activity. The evoked power is simply the power of the input, because the noise and background terms are suppressed by averaging.

$$
\begin{aligned}
g(\omega, t)_{e} & =|\Gamma(\omega, t)|^{2}\left\langle s(\omega, t)_{\alpha}\right\rangle\left\langle s(\omega, t)_{\alpha}{ }^{*}\right\rangle \\
& =|\Gamma(\omega, t)|^{2} g(\omega, t)_{\alpha}
\end{aligned}
$$

The baseline power at $t=t_{0}$ is

$g(\omega)_{b}=\left|\Gamma\left(\omega, t_{0}\right)\right|^{2} g(\omega)_{\beta}+g(\omega)_{\varepsilon}$

This means that induced power is

$g(\omega, t)_{i}=\left(|\Gamma(\omega, t)|^{2}-\left|\Gamma\left(\omega, t_{0}\right)\right|^{2}\right) g(\omega)_{\beta}$

This is an important result. It means that the only way induced power can be expressed is if the transfer function $\Gamma(\omega, t, \theta)$ changes at time $t$. This can only happen if the parameters of the neuronal system change. In other words, only structural effects can mediate induced power. However, this does not mean to say that structural effects are expressed only in induced power. They can also be expressed in the evoked power: Eq. (13) shows clearly that evoked power at a particular point in peristimulus time depends on both $g(\omega, t)_{\alpha}$ and $\Gamma(\omega, t, \theta)$. This means that structural effects mediated by changes in the transfer function can be expressed in evoked power, provided $g(\omega, t)_{\alpha}>0$. In other words, structural effects can modulate the expression of stationary components due to ongoing activity and also deterministic components elicited dynamically. To summarize so far:

- Dynamic effects (of driving inputs) conform to a generalized convolution of inputs to form the systems response.

- Structural effects can be formulated as a time-dependent change in the parameters (that may be mediated by modulatory inputs). This translates into time-dependent change in the convolution kernels and ensuing response.

- If the ongoing activity is nonzero and stationary, only structural effects can mediate induced power.

- If stimulus-related input is nonzero, structural effects can also mediate evoked power, i.e., dynamic and structural effects can conspire to produce evoked power.
In the next section, we demonstrate these theoretical considerations in a practical setting, using a neural mass model of event-related responses. In this section and in the simulations below, we have only considered effect of a single trial type. In practice, one would normally compare the responses evoked and induced by two trial types. However, the conclusions are exactly the same in both contexts. One can regard the simulations below as a comparison of one trial type to a baseline that caused no response (and had no baseline power).

\section{Modeling induced oscillations}

\section{Neural mass models}

The classical approach to modeling MEG/EEG signals is to use neural mass models (Freeman, 1978; Lopes da Silva et al., 1974; Robinson et al., 2001). The idea is to model the state of a neuronal assembly, i.e., thousands of identical neurons, using operations that describe the mean input-output relationships. For example, one can summarize the state of a neuronal assembly with its mean membrane potential and firing rate. The expected potential can be obtained using a linear convolution of the mean firing rate with a gamma function. This function can be understood as the postsynaptic potential impulse response function. The output of the neuronal assembly is a mean firing rate, which is a nonlinear (sigmoid) function of the mean membrane potential. There are several models of MEG activity that are based upon this approach. In particular, the Jansen model (Jansen and Rit, 1995) mimics the canonical architecture of a mini-column, which can be treated as a cortical source. The Jansen model comprises three neuronal populations: excitatory and inhibitory interneurons and pyramidal cells. The MEG/EEG signal is assumed to be proportional to the depolarization of pyramidal cells. The parameters of the model are the synaptic time constants, efficacies and coupling parameters that control the intrinsic connections within a source and extrinsic connections among sources. This model and various extensions have been used to simulate oscillatory activity (David and Friston, 2003; David et al., 2004; Jansen and Rit, 1995), evoked responses (Jansen and Rit, 1995; David et al., 2005) and epileptic activity (Wendling et al., 2000).

Here, we model MEG/EEG signals using the Jansen model (Jansen and Rit, 1995), extended to cover neuronal ensembles with different kinetics (David and Friston, 2003) and extrinsic corticocortical connections (David et al., 2005; Crick and Koch, 1998). This model defines the state equation and observer in Eq. (1). The details of these equations are not important in the present context. A detailed description of the model can be found in David et al. (2005) and the Matlab scripts are also available to download from www.fil.ion.ucl.ac.uk/spm (as part of the DCM for ERPs toolbox; e.g., spm_erp_fx.m).

The model used here does not model explicitly the diversity of neuronal subpopulations and their processes, such as glial-neuron interactions. However, because the Jansen model is a lumped representation of diverse processes, one explicit parameter, such as coupling between regions, can be understood as representing diverse phenomena. For instance, when we manipulate coupling parameters, we explicitly modify the efficacy of cortico-cortical connections. The neural mechanisms responsible for this increase of connection strength are diverse: modulation of transmitter release, modulation of local synchronization due to glial cells, etc. These relatively fine-scale processes are not included in the Jansen 
model, but the mean field approximation of neural mass models can, in many instances, capture the dynamics that emerge. We chose Jansen's neural mass model of EEG because there has been a considerable amount of work showing that it can reproduce most of the phenomena and dynamics seen in real EEG data.

The key thing is that the model has a relatively high degree of face validity, when it comes to modeling neuronal dynamics and, critically, allows selective dynamic or structural perturbation.

We consider a simple model composed of two sources, interconnected with forward and backward connections (Fig. 1B). The sources receive two types of inputs. The first models afferent activity that delivers dynamic perturbations to the systems states (by changing postsynaptic currents). This dynamic perturbation had stochastic and deterministic components: background inputs $\beta^{(i)}$ comprised Gaussian noise that was delivered to both sources. The deterministic part modelled a stimulus with an impulse $\alpha(t)=\delta(0)$, delivered to the first source at the beginning of each trial. The second sort of input $u(t)_{M}$ induced a structural change by modulating extrinsic connections. As one might expect, the effects of these two input classes differ considerably. On the one hand, synaptic inputs perturb the system nearly instantaneously, and the deterministic part evokes responses that are phase locked to the stimulus. On the other hand, modulatory inputs modify the manifold that attracts ongoing activity, without necessarily resetting its phase. For simplicity, we restrict our modulatory effects to a modulation of the extrinsic backward connection, thus encompassing various synaptic mechanisms which modify the gain of excitatory synapses (Salinas and Their, 2000). We chose this form of modulation because it was the simplest. Structural perturbations change the systems parameters (i.e., coupling parameters modeling synaptic efficacy). We elected to change one extrinsic connection; the backward connection. We chose the backward connection because backward connections are associated with modulatory effects, both in terms of physiology (e.g., the mediation of extraclassical receptive field effects, see also Allman et al., 1985 and Murphy et al., 1999) and anatomy (e.g., they terminate in supragranular layers that expressed large number of voltage-dependent NMDA receptors). See also Maunsell and van Essen (1983) and Angelucci et al. (2002).

There may be many other modulatory mechanisms that will produce the same pattern of oscillatory activity, and it will be an interesting endeavor to disambiguate the locus of structural changes using these sorts of models and empirical data (see David et al., 2005).

\section{Structural perturbation and induced oscillations}

We assume that the stimulus engages a cascade of neural events, involving recurrent hierarchical dynamics that modulates the systems structure. This is modeled by the deterministic modulatory input $u(t)_{M}$. In our model (Fig. 1B), this increases the strength of backward connections between the sources. This could be regarded as modeling voltage dependence in a massive pool of NMDA receptors located in the supragranular targets of backward connections, although many other mechanisms could be approximated by such changes in connectivity. In this example, we use the following structural perturbation.

$$
\begin{aligned}
& \theta(t)_{B}=\theta_{B}\left(1+u(t)_{M}\right) \\
& u(t)_{M}= \begin{cases}\frac{t-t_{0}}{\tau} \exp \left(-\frac{\left(t-t_{0}\right)}{\tau}\right) & t \geq t_{0} \\
0 & t<t_{0}\end{cases}
\end{aligned}
$$

where $t_{0}$ and $\tau$ are the onset and time constant of the modulating input, respectively. This input acts on the parameter $\theta_{B}$ of the state equation to smoothly modify the convolution of ongoing and driving inputs.

To illustrate the points of the previous section, we will consider two scenarios in which the modulatory effect arrives at the same time as the driving input and one in which it arrives after the dynamic perturbation has dissipated. Let us assume that the modulatory input has a slow time constant $\tau=150 \mathrm{~ms}$ compared to the main frequency of ongoing oscillations $(10 \mathrm{~Hz})$. The modulatory effects can be expressed with stimulus onset or after some delay. In the first case, evoked oscillations will be modulated, and these effects will be visible in the ERR. In the second case, phase locking with the stimulus will have been lost, and no effect will be seen in the ERR. However, in both cases, structural changes will appear as induced oscillations. This is illustrated in Fig. 2 (using 500 trial averages). In the upper panel we consider a modulatory input immediately after stimulus onset. As expected, evoked responses are much more pronounced relative to delayed modulation (lower panel). The induced power (C) shows that increases in the backward connection induce oscillations in the alpha and gamma band. The induced power in Fig. 2 has been frequency normalized (by removing the mean and dividing by the standard deviation at $t=0$ ) to show increased power in the gamma band more clearly.

These simulations provide a nice model for induced responses using a structural perturbation, in this instance, a slow modulation of the efficacy of backward connections in a simple hierarchy of neuronal populations. Critically, these simulations also show that responses can be evoked structurally by a modulation of dynamic perturbations. This dual mechanism depends on driving and modulatory effects occurring at the same time, causing evoked and induced responses in the same time-frequency window.

\section{Structurally evoked responses and phase resetting}

Phase resetting is a popular perspective on mechanisms responsible for evoked responses (David et al., 2005; Makeig et al., 2002; Klimesch et al., 2004). Phase resetting is inferred when there is a phase locking of responses, with no change in their amplitude. It is tempting to formulate phase resetting in terms of dynamic and structural mechanisms and, in particular, the appearance of evoked responses that are mediated by structural mechanisms, as in Fig. 2. We have argued previously (David et al., 2005) that phase resetting entails an interaction between the input and the neuronal state of an ensemble (e.g. the phase of ongoing oscillations). The response component $u(t)_{M} \partial J / \partial u(t)_{M} x$ in Eq. (5) embodies this interaction. This means phase resetting is a structural effect that is mediated by a deterministic, modulatory, component of the input.

However, the joint expression of evoked and induced responses does not imply phase resetting. This is because phase resetting is a very specific structural mechanism that entails a reduction of induced power. The reason is simple; if the stimulus does not change the amplitude of oscillations, the total power will be constant over peristimulus time. Phase resetting will increase evoked power. Because induced power is the total power that cannot be explained by evoked and baseline power, it must fall. This provides the basis for a test for phase resetting, which we will pursue in a later communication. Note that analyses framed in terms of phase-distributions over trials (i.e., phase resetting analyses) 
Fig. 2. Upper panel: simulation of fast stimulus-related modulation of backward connectivity, using the model depicted in Fig. 1B. Black curves are the responses of area 1; grey curves correspond to area 2. Time-frequency responses are shown for area 1 only. The white line, superimposed on these spectral profiles, shows the time course of the modulatory input. (A) Evoked power, after averaging over trials, showing late oscillations that have been augmented by modulatory input. (B) Total Power, averaged over trials. (C) Induced power, normalized over frequency. Lower Panel: As for the upper panel, but here the modulatory effect has been delayed. The main difference is that low-frequency evoked components have disappeared because dynamic and structural perturbations are now separated in time and cannot interact. See main text for further details.

discount amplitude variations and assume that differences in phase distributions are not mediated by amplitude differences.

Having established that evoked responses can be mediated by structural mechanisms, we now show that induced responses can be mediated by dynamic mechanisms.

\section{Induced oscillations and trial-to-trial variability}

Above, we have considered the stimulus as a deterministic input. In this section, we consider what would happen if the stimulus-related input was stochastic. This randomness is most easily understood in terms of trial-to-trial variability in the inputs. Following Truccolo et al. (2002), we examine two random aspects of inputs, namely stochastic variations in gain and latency. We derive equations that predict the effects of this variability on evoked and induced responses, and we test the predictions using the model of the previous section.

\section{Trial-to-trial variability}

As suggested in Truccolo et al. (2002), we consider two types of variability in the input. The first relates to a trial-to-trial gain or amplitude variations. For an identical stimulus, early processing may introduce variations in the amplitude of driving inputs to a source. Gain modulation is a ubiquitous phenomenon in the central nervous system (Salinas and Their, 2000), but its causes are not completely understood. Two neurophysiological mechanisms that may mediate gain modulation include fluctuations of extracellular calcium concentration (Smith et al., 2002) and/or of the overall level of synaptic input to a neuron (Chance et al., 2002). These may act as a gain control signal that modulates responsiveness to excitatory drive. A common example of gain effects, in a psychophysiological context, is the effect of attention (McAdams and Maunsell, 1999; Treue and Martinez-Trujillo, 1999).

The second commonly observed source of variability is in the latency of input onset, i.e., the time between the presentation of the stimulus and the peak response of early processing. Azouz and Gray (1999) have investigated the sources of such latency variations at the neuronal level. Basically, they describe two major phenomena: (i) coherent fluctuations in cortical activity preceding the onset of a stimulus have an impact on the latency of neuronal responses (spikes). This indicates that the time needed to integrate activity to reach action potential threshold varies between trials. (ii) The other source of latency variability is fluctuations in the action potential threshold itself.

Both types of trial-to-trial variability, gain modulation and latency, can be modeled by introducing the random variables $\gamma$ and $\tau$ with density functions $p(\gamma)$ and $p(\tau)$. In the context of random latencies, the expected Fourier transform of the stimulus-related 
component is modulated by the Fourier transform $s(\omega)_{\tau}$ of the probability density $p(\tau)^{3}$

$$
\begin{aligned}
& s(\omega, t, \tau)_{\alpha}=\int k(\omega, \sigma) \alpha(t-\sigma-\tau) d \sigma \\
& \left\langle s(\omega, t, \tau)_{\alpha}\right\rangle=\iint p(\tau) k(\omega, \sigma) \alpha(t-\sigma-\tau) d \sigma d \tau \\
& =\iint p(\tau) \exp (j \omega \tau) k(\omega, \sigma-\tau) \alpha(t-\sigma-\tau) d \sigma d \tau \\
& =\int p(\tau) \exp (j \omega \tau) d \tau \int k(\omega, \sigma-\tau) \alpha(t-\sigma-\tau) d \sigma \\
& =s(\omega)_{\tau} s(\omega, t)_{\alpha}
\end{aligned}
$$

This is known as the characteristic function

$$
s(\omega)_{\tau}=\int p(\tau) \exp (j \omega \tau) d \tau
$$

A very tight latency distribution makes $g(\omega)_{\tau}=s(\omega)_{\tau} s(\omega)_{\tau} *$ very broad over frequency and its effect is negligible. However, if the latencies are more dispersed the modulation by the characteristic function become tighter with a suppression of high frequencies. In terms of evoked responses.

$$
\begin{aligned}
g(\omega, t)_{e} & =|\Gamma(\omega, t)|^{2}\left\langle s(\omega, t, \tau)_{\alpha}\right\rangle\left\langle s(\omega, t, \tau)_{\alpha}^{*}\right\rangle \\
& =|\Gamma(\omega, t)|^{2} g(\omega)_{\tau} g(\omega, t)_{\alpha}
\end{aligned}
$$

This equality shows that high-frequency components are lost when latency varies randomly over trials. This means that ERR will be estimated badly at high frequencies. This variation effectively blurs or smoothes the average and suppresses fast oscillations in the evoked response. However, the total power remains unchanged because the power expressed in each trial does not depend on latency. Therefore, the high frequencies lost from the evoked responses now appear in the induced response.

$g(\omega, t)_{i}=\left(|\Gamma(\omega, t)|^{2}-\left|\Gamma\left(\omega, t_{0}\right)\right|^{2}\right) g(\omega)_{\beta}+\eta g(\omega, t)_{e}$

$\eta=\frac{1-g(\omega)_{\tau}}{g(\omega)_{\tau}}$

In summary, the induced power has now acquired a stimuluslocked component. This component gets bigger as the dispersion of latencies increases and $g(\omega)_{\tau}$ gets smaller. Note that this dynamically induced power can only be expressed in frequencies that show evoked responses, because both depend on $g(\omega, t)_{\alpha}$, the power in the stimulus-locked input.

A similar analysis can be pursued for variations in gain. Here we will assume, by definition $\langle\gamma\rangle=1$.

$$
\begin{aligned}
& s(\omega, t, \gamma)_{\alpha}=\gamma s(\omega, t)_{\alpha} \\
& \left\langle s(\omega, t, \gamma)_{\alpha}\right\rangle\left\langle s(\omega, t, \gamma)_{\alpha}^{*}\right\rangle=\langle\gamma\rangle^{2} g(\omega, t)_{\alpha}=g(\omega, t)_{\alpha} \\
& \left\langle s(\omega, t, \gamma)_{\alpha} s(\omega, t, \gamma)_{\alpha}^{*}\right\rangle=\left\langle\gamma^{2}\right\rangle g(\omega, t)_{\alpha}
\end{aligned}
$$

\footnotetext{
${ }^{3}$ We have assumed here and below that the variation in latency is small in relation to the length of the wavelet used in the time-frequency decomposition.
}

Giving

$$
\begin{aligned}
& g(\omega, t)_{i}=\left(|\Gamma(\omega, t)|^{2}-\left|\Gamma\left(\omega, t_{0}\right)\right|^{2}\right) g(\omega)_{\beta}+\eta g(\omega, t)_{e} \\
& \eta=\operatorname{Var}(\gamma)
\end{aligned}
$$

Gain variations also allow nonstructural mechanisms to induce power. Here, the time-dependent changes in stimulus-dependent power $g(\omega, t)_{\alpha}$ again contribute to induced responses. In this instance the contribution is not frequency specific, as with latency variations, but proportional to the variance in gain $\operatorname{Var}(\gamma)=\left\langle\gamma^{2}\right\rangle-$ 1. To summarize:

- Induced power can be mediated by nonstructural mechanisms if dynamic responses are caused by inputs that vary over trials.

- Latency variations in stimulus-locked inputs effectively suppress high frequencies in the average that are effectively transferred from the evoked power to the induced power.

- Amplitude variations in stimulus-locked inputs do not affect evoked responses but cause evoked power to be recapitulated in the induced power as the variance of the amplitude increases.

We now illustrate these phenomena using simulations.

\section{Simulations of dynamically induced responses}

To ensure that any induced power in the simulations could not be mediated structurally, we removed both the modulatory and stochastic input. Therefore, there were no structurally mediated changes in the systems manifold or kernels, and, even if there were, they would not be seen because there was no ongoing activity. This means that any induced power must be cased dynamically. 2000 trials were simulated with variations in latency and gain respectively.

First we simulated a pure latency jittering, without gain modulation (Fig. 3). The stimulus onset latency $p(\tau)=N\left(0, \sigma_{\tau}^{2}\right)$ was sampled from a Gaussian distribution with zero mean and standard deviation $\sigma_{\tau}=10 \mathrm{~ms}$. The upper left panel shows the event-related and evoked responses for a single trial. The black curve is the response of area 1; the grey curve is the response of area 2 . These responses would constitute the ERR, after averaging, without trial-totrial variability. The time-frequency decomposition of the response of area 1 shows two blobs; one located in the alpha band (around 10 $\mathrm{Hz}$ ), the other in the gamma band (around $30 \mathrm{~Hz}$ ). These correspond to the responses of specific neuronal subpopulations. Fig. 3B shows the event-related and evoked responses after averaging over all trials. Latency variation causes evoked power to be lost at high frequencies, as it is smoothed away in the average (compare the evoked responses in panel $\mathrm{B}$ with the single-trial or total power in panels $\mathrm{A}$ and $\mathrm{C}$ ). The lost power now appears in the induced responses, more markedly at higher frequencies (gamma band in Fig. 3D). This dynamically induced response has to occur at the same time as the evoked response but is expressed in higher frequencies.

Finally, variation in gain was simulated. The gain $\gamma$ for each trial was drawn from a $\log$ normal distribution $p(\ln \lambda)=N(0,0.36)$. Fig. 4 summarizes the results of these simulations using the same format as the previous figure. Fig. 4B shows the event-related and evoked responses after averaging over all trials. As expected, there is no significant difference between these evoked responses and the canonical, single-trial response in Fig. 4A. However, the induced power (D) is not zero and, as predicted, formally very similar to the evoked- 

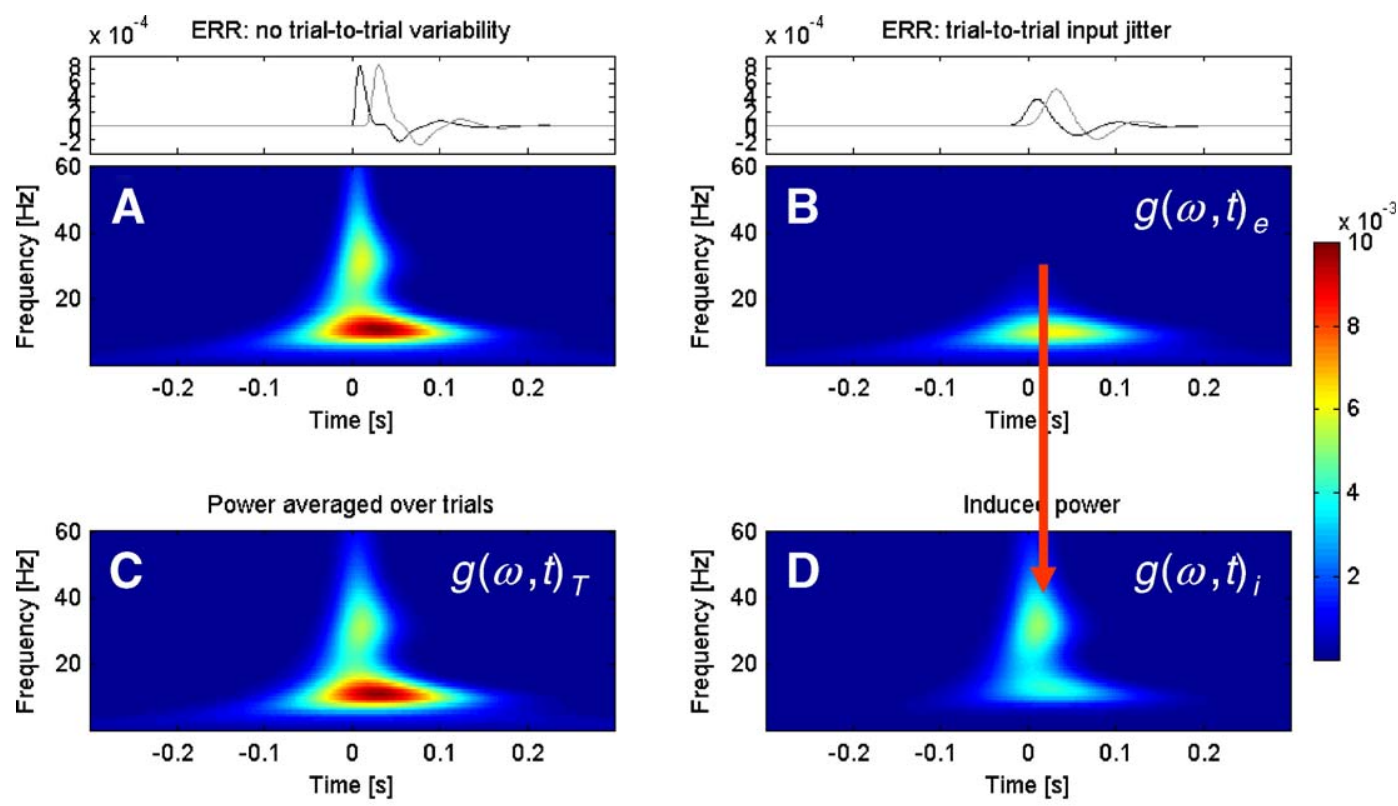

Transfer of energy from evoked to induced responses

Fig. 3. Simulation of trial-to-trial latency jitter, using the model depicted in Fig. 1B. Black curves are the responses of area 1; grey curves correspond to area 2. Time-frequency responses are shown for area 1 only. (A) Canonical response to a stimulus at time zero. (B) Evoked responses, after averaging over trials. (C) Total power, averaged over trials. (D) Induced power. As predicted, high-frequency induced oscillations emerge with latency jittering (D). This is due to the fact that trial-averaging removes high frequencies from the evoked power; as a result, they appear in the induced response.

power. Critically, the induced and evoked responses generated by this mechanism have the same time-frequency deployment.

\section{Discussion}

\section{Summary}

In summary, we made a distinction between dynamic and structural mechanisms that underlie transient responses to perturbations.
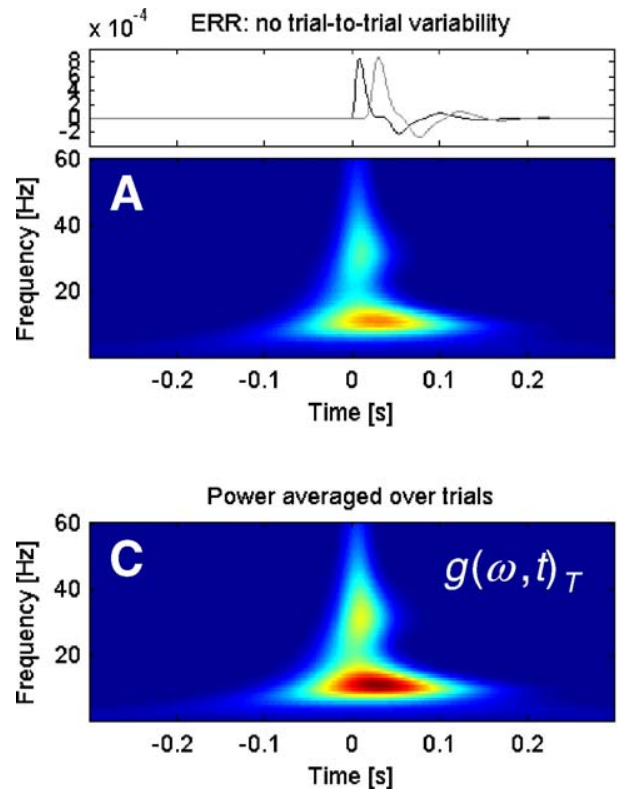

We then considered how responses are measured in time-frequency in terms of evoked and induced responses. Theoretical predictions, confirmed by simulations, show that there is no simple relationship between the two mechanisms causing responses and the two ways in which they are characterized. Specifically, evoked responses can be mediated both structurally and dynamically. Similarly, if there is trial-to-trial variability, induced responses can be mediated by both mechanisms. See Fig. 5 for a schematic summary.

For evoked responses, this is not really an issue. The fact that evoked responses reflect both dynamic and structural perturbations

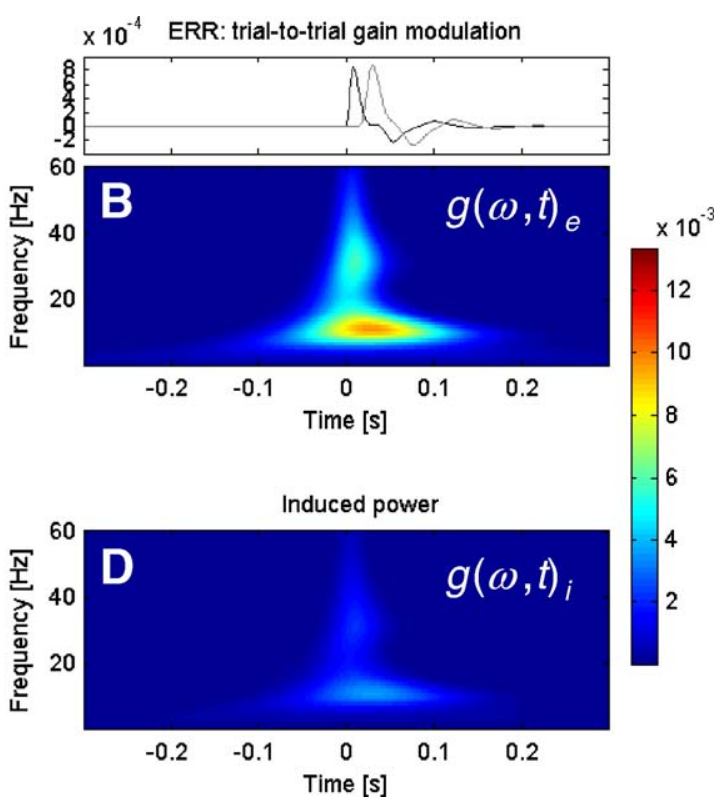

Fig. 4. Simulation of gain variations over trials. The format is the same as in Fig. 3. As predicted, although gain variation has no effect on evoked power it does affect induced power, rendering it a 'ghost' of the evoked power. See main text for details. 


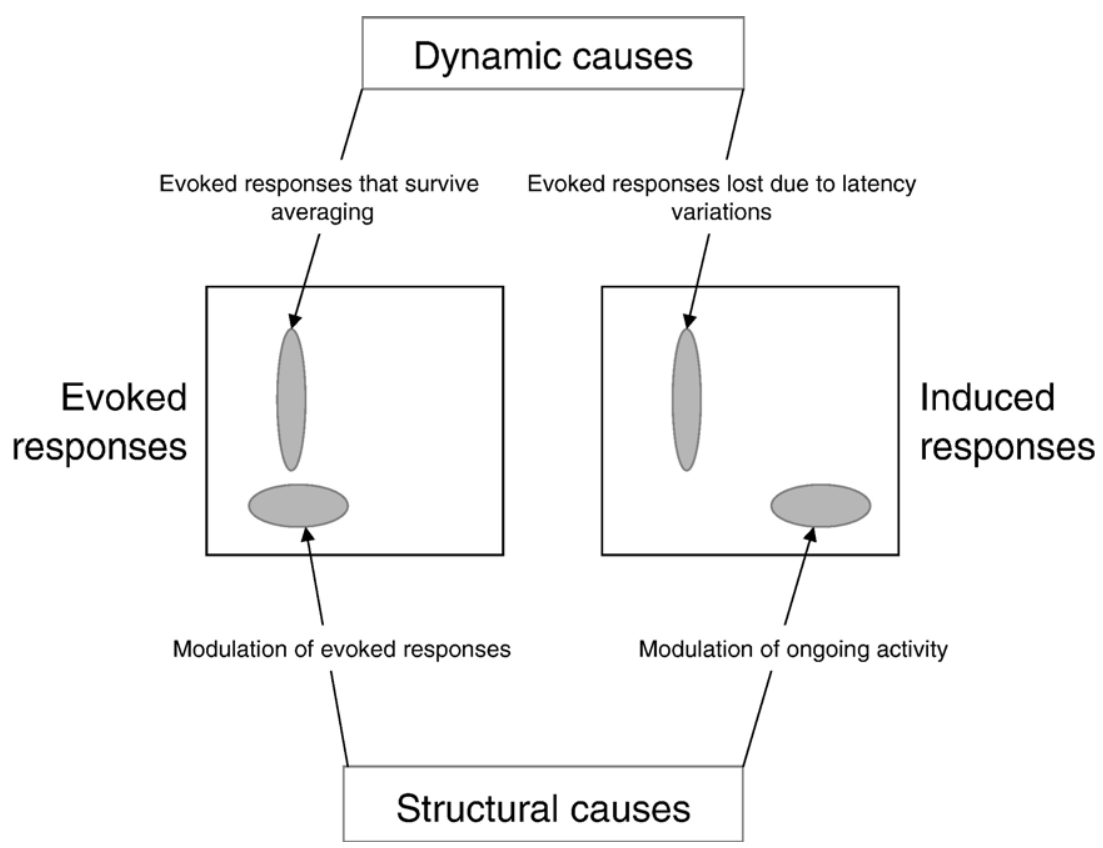

Fig. 5. Schematic illustrating the many-to-many mapping between dynamic vs.. structural causes and evoked vs. induced responses.

is sensible, if one allows for the fact that any input can have dynamic and structural effects. In other words, the input perturbs the states of the neuronal system and, at the same time, modulates interactions among the states. The structural component here can be viewed as a nonlinear (e.g., bilinear) effect that simply involves interactions between the input and parameters (e.g., synaptic status). Generally, the structurally mediated component of evoked responses will occur at the same time and frequency as the dynamically mediated components. This precludes ambiguity when interpreting evoked responses, if one allows for both dynamic and structural causes.

The situation is more problematic for induced responses. In the absence of trial-to-trial variability induced responses must be caused by structural perturbations. Furthermore, there is no necessary co-localization of evoked and induced responses in time-frequency because induced responses are disclosed by ongoing activity. However, if trial-to-trial variability is sufficient, induced responses with no structural component will be expressed. This means that induced responses that occur at the same time as evoked responses have an ambiguity in relation to their cause. Happily, this can be addressed at two levels. First, induced responses that do not overlap in peristimulus time cannot be attributed to dynamic mechanisms and are therefore structural in nature. Second, one can revisit the operational definition of induced responses to derive a measure that is immune to the effects of trialto-trial variability. Note that here we do not consider that the baseline activity is affected directly by the stimulus but interacts with stimulus-dependent structural mechanisms to produce an induced response component. Clearly, this component will then form the input to other regions.

\section{Adjusted power}

In this subsection, we introduce the notion of adjusted power as a complementary characterization of structurally mediated responses. Adjusted power derives from a slightly more explicit formulation of induced responses as that component of total power that cannot be explained by evoked or ongoing activity. The adjusted response is simply the total power orthogonalized, at each frequency, with respect to baseline and evoked power.

$g(\omega, t)_{a}=g(\omega, t)_{T}-g(\omega, t) \hat{\eta}$

$\hat{\eta}=g(\omega, t)^{+} g(\omega, t)_{T}$

$g(\omega, t)=\left[\begin{array}{cc}1 & g\left(\omega, t_{1}\right)_{e} \\ \vdots & \vdots \\ 1 & g\left(\omega, t_{T}\right)_{e}\end{array}\right]$

+ denotes the pseudoinverse. Note that the evoked power has been augmented with a constant that models baseline power. This means baseline power does not have to be estimated explicitly. The motivation for this linear adjustment is inherent in Eqs. (20) and (22), which show that the confounding effects of trial-to-trial variability are expressed in proportion to evoked power. Eq. (23) is implicitly estimating baseline power and the contribution from evoked power and removing them from the total power. In other words, $\eta$ is a 2 -vector estimate of $g(\omega)_{b}$ and $(1+\eta)$. After these components have been removed, the only components left must be structural in nature

$g(\omega, t)_{a}\left(|\Gamma(\omega, t)|^{2}-\left|\Gamma\left(\omega, t_{0}\right)\right|^{2}\right) g(\omega)_{\beta}$

Fig. 6 shows that the effect of trial-to-trial variability on induced responses disappears when using adjusted power. This means one can unambiguously attribute adjusted responses to structural mechanisms. As noted by one of our reviewers the ERPadjusted response removes evoked response components, including those mediated by structural changes. However, structurally mediated induced components will not be affected unless they have the same temporal expression. The usefulness of adjusted power, in an empirical setting will be addressed in future work. The treatment in this paper can be regarded as establishing its motivation. 

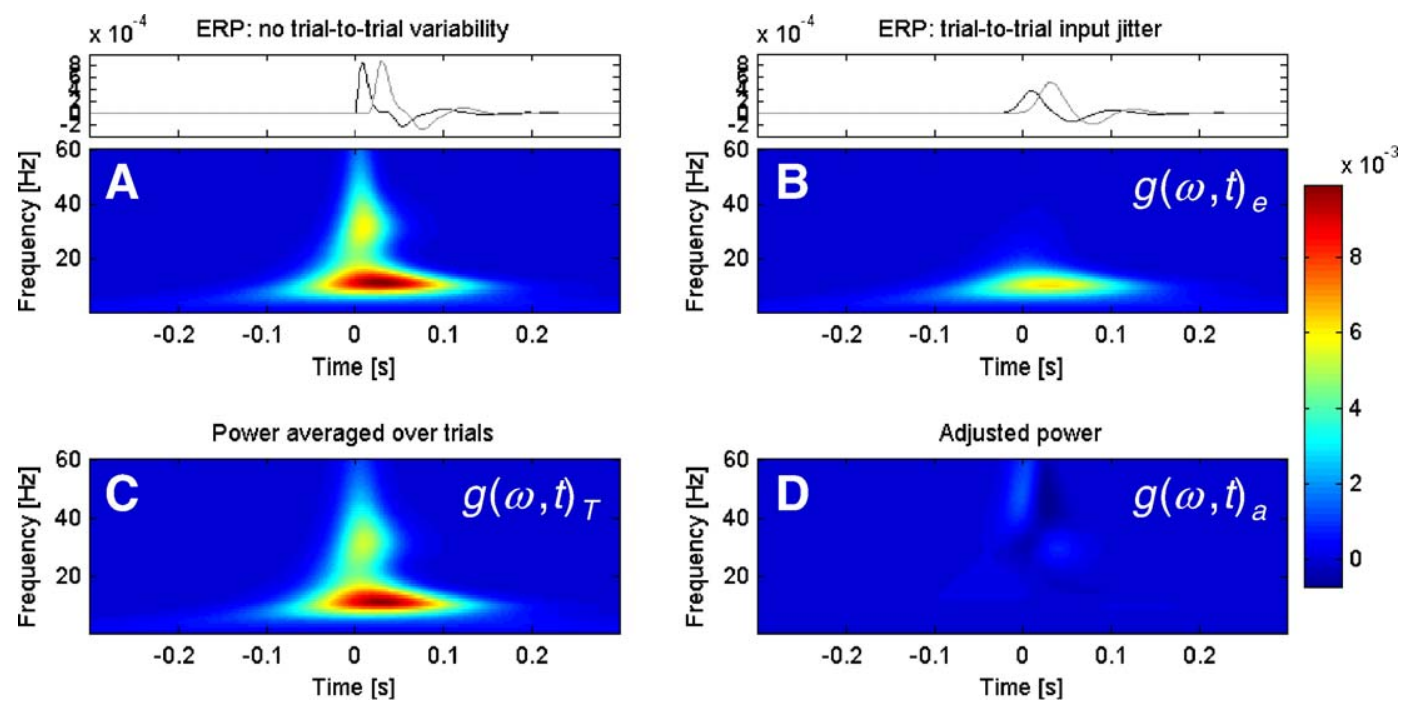

Fig. 6. Adjusted power (3D). The format is the same as in Fig. 3. As predicted, the adjusted power is largely immune to the effects of latency variation, despite the fact that evoked responses still lose their high-frequency components.

\section{Conclusion}

We have divided neuronal mechanisms into dynamic and structural, which may correspond to driving and modularity neurotransmitter systems respectively. These two sorts of effects are not equivalent to evoked and induced responses in MEG/EEG. By definition, evoked responses exhibit phase locking to a stimulus whereas induced responses do not. Consequently, averaging over trials discounts both ongoing and induced components and evoked responses are defined by the response averaged over trials. Evoked responses may be mediated primarily by driving inputs. In MEG/ EEG, driving inputs affect the state of measured neuronal assemblies, i.e., the dendritic currents in thousands of pyramidal cells. In contradistinction, structural effects, mediated by modulatory inputs, engage neural mechanisms which affect neuronal states, irrespective of whether they are phase locked to the stimulus or not. These inputs are expressed formally as time-varying parameters of the state equations modeling the systems. Although the ensuing changes in the parameters may be slow and enduring, their effects on ongoing or evoked dynamics may be expressed as fast or highfrequency dynamics.

We have considered a further cause of induced oscillations, namely trial-to-trial variability of driving inputs. As suggested in Truccolo et al. (2002), these can be modeled by varying latency and gain. We have shown that (i) gain variations have no effect on the ERR but increase induced responses in proportion to evoked responses, (ii) jitter in latency effectively smoothes the evoked responses and transfers energy from evoked to induced power, preferentially at higher frequencies.

The conclusions of this work, summarized in Fig. 5, provide constraints on the interpretation of evoked and induced responses in relation to their mediation by dynamic and structural mechanisms. This is illustrated by the work of Tallon-Baudry and colleagues, who have looked at non-phase-locked episodes of synchronization in the gamma-band $(30-60 \mathrm{~Hz})$. They have emphasized the role of these induced responses in feature-binding and top-down mechanisms of perceptual synthesis. The top-down aspect is addressed by their early studies of illusory perception (Tallon-Baudry et al., 1996), where the authors "tested the stimulus specificity of highfrequency oscillations in humans using three types of visual stimuli: two coherent stimuli (a Kanizsa and a real triangle) and a noncoherent stimulus." They found an early phase locked $40-\mathrm{Hz}$ component, which did not vary with stimulation type and a second $40-\mathrm{Hz}$ component, appearing around $280 \mathrm{~ms}$, that was not phase locked to stimulus onset. This shows a nice dissociation between early evoked and late induced responses. The induced component was stronger in response to a coherent triangle, whether real or illusory and "could reflect, therefore, a mechanism of feature binding based on high-frequency synchronization". Because it was late, the induced response can only be caused by structural mechanisms (see Fig. 5). This is consistent with the role of topdown influences and the modulatory mechanisms employed by backward connections in visual synthesis (Maunsell and van Essen, 1983; Bullier et al., 2001; Albright and Stoner, 2002).

Classical ERP/ERF research has focused on dynamic perturbations (Coles and Rugg, 1995). On the other hand, studies of eventrelated synchronization (ERS) or desynchronization (ERD) are more concerned with structural effects that may be mediated by modulatory systems (Pfurtscheller and Lopes da Silva, 1999). Practically speaking, we have shown that it is not always possible to distinguish between dynamic and structural effects when inferring the causes of evoked and induced oscillations. However, certain features of induced oscillations might provide some hints: (i) induced oscillations in high frequencies concomitant with evoked responses in low frequencies may indicate a jittering of inputs. (ii) Induced oscillations that are temporally dissociated from evoked responses are likely to be due to modulatory or structural effects. Finally, we have introduced the notion of adjusted power that can be unambiguously associated with structural effects.

\section{Acknowledgment}

The Wellcome trust funded this work.

\section{References}

Albright, T.D., Stoner, G.R., 2002. Contextual influences on visual processing. Annu. Rev. Neurosci. 25, 339-379.

Allman, J., Miezin, F., McGuinness, E., 1985. Stimulus specific responses 
from beyond the classical receptive field: neurophysiological mechanisms for local-global comparisons in visual neurons. Annu. Rev. Neurosci. 8, 407-430.

Angelucci, A., Levitt, J.B., Lund, J.S., 2002. Anatomical origins of the classical receptive field and modulatory surround field of single neurons in macaque visual cortical area V1. Prog. Brain Res. 136, 373-388.

Azouz, R., Gray, C.M., 1999. Cellular mechanisms contributing to response variability of cortical neurons in vivo. J. Neurosci. 19 (6), 2209-2223.

Breakspear, M., Terry, J.R., 2002. Detection and description of non-linear interdependence in normal multichannel human EEG data. Clin. Neurophysiol. 113, 735-753.

Breakspear, M., Terry, J.R., Friston, K.J., 2003. Modulation of excitatory synaptic coupling facilitates synchronization and complex dynamics in a biophysical model of neuronal dynamics. Network 14, 703-732.

Bullier, J., Hupe, J.M., James, A.C., Girard, P., 2001. The role of feedback connections in shaping the responses of visual cortical neurons. Prog. Brain Res. 134, 193-204.

Chance, F.S., Abbott, L.F., Reyes, A.D., 2002. Gain modulation from background synaptic input. Neuron 35 (4), 773-782.

Coles, M.G.H., Rugg, M.D., 1995. Event-related brain potentials: an introduction. In: Rugg, M.D., Coles, M.G.H. (Eds.), Electrophysiology of Mind. Oxford Univ. Press, Oxford, pp. 1-26.

Crick, F., Koch, C., 1998. Constraints on cortical and thalamic projections: the no-strong-loops hypothesis. Nature 391, 245-250.

David, O., Friston, K.J., 2003. A neural mass model for MEG/EEG: coupling and neuronal dynamics. NeuroImage 20, 1743-1755.

David, O., Cosmelli, D., Friston, K.J., 2004. Evaluation of different measures of functional connectivity using a neural mass model. NeuroImage 21, 659-673.

David, O., Harrison, L., Friston, K.J., 2005. Modelling event-related responses in the brain. NeuroImage 25, 756-770.

David, O., Kiebel, S.J., Harrison, L.M., Mattout, J., Kilner, J.M., and Friston, K.J., in press. Dynamic causal modelling of evoked responses in EEG and MEG. NeuroImage.

Freeman, W.J., 1978. Models of the dynamics of neural populations. Electroencephalogr. Clin. Neurophysiol. 34, 9-18 ((Suppl)).

Friston, K.J., 1997. Transients metastability and neuronal dynamics. NeuroImage 5, 164-171.

Friston, K.J., 2001. Brain function, nonlinear coupling, and neuronal transients. Neuroscientist 7 (5), 406-418 ((Oct)).

Galambos, R., 1992. In: Basar, E., Bullock, T.H. (Eds.), Induced Rhythms in the Brain. Birkhauser, pp. 201-216.

Jansen, B.H., Rit, V.G., 1995. Electroencephalogram and visual evoked potential generation in a mathematical model of coupled cortical columns. Biol. Cybern. 73, 357-366.

Kiebel, S.J., Tallon-Baudry, C., Friston, K.J., 2005. Parametric analysis of oscillatory activity as measured with EEG/MEG. Hum. Brain Mapp. 26, $170-177$.

Klimesch, W., Schack, B., Schabus, M., Doppelmayr, M., Gruber, W., Sauseng, P., 2004. Phase-locked alpha and theta oscillations generate the P1-N1 complex and are related to memory performance. Brain Res. Cogn. Brain Res. 19, 302-316.

Lopes da Silva, F.H., Hoeks, A., Smits, H., Zetterberg, L.H., 1974. Model of brain rhythmic activity. The alpha-rhythm of the thalamus. Kybernetik 15, 27-37.

Makeig, S., Westerfield, M., Jung, T.P., Enghoff, S., Townsend, J., Courchesne, E., Sejnowski, T.J., 2002. Dynamic brain sources of visual evoked responses. Science 295, 690-694.

Maunsell, J.H., van Essen, D.C., 1983. The connections of the middle temporal visual area (MT) and their relationship to a cortical hierarchy in the macaque monkey. J. Neurosci. 3, 2563-2586.

McAdams, C.J., Maunsell, J.H.R., 1999. Effects of attention on orientationtuning functions of single neurons in macque cortical area V4. J. Neurosci. 19 (431), 441.

Murphy, P.C., Duckett, S.G., Sillito, A.M., 1999. Feedback connections to the lateral geniculate nucleus and cortical response properties. Science 286, $1552-1554$.

Pfurtscheller, G., Lopes da Silva, F.H., 1999. Event-related EEG/MEG synchronization and desynchronization: basic principles. Clin. Neurophysiol. 110, $1842-1857$.

Robinson, P.A., Rennie, C.J., Wright, J.J., Bahramali, H., Gordon, E., Rowe, D.L., 2001. Prediction of electroencephalographic spectra from neurophysiology. Phys. Rev., E 63, 021903.

Salinas, E., Their, P., 2000. Gain modulation: a major computation principle of the central nervous system. Neuron 27, 15-21.

Sherman, S.M., Guillery, R.W., 1998. On the actions that one verve cell can have on another: distinguishing "drivers" from "modulators". Proc. Nat. Acad. U. S. A. 95, 7121-7126.

Singer, W., Gray, C.M., 1995. Visual feature integration and the temporal correlation hypothesis. Annu. Rev. Neurosci. 18, 555-586.

Smith, M.R., Nelson, A.B., Du Lac, S., 2002. Regulation of firing response gain by calcium-dependent mechanisms in vestibular nucleus neurons. J. Neurophysiol. 87, 2031-2042.

Stam, C.J., Pijn, J.P.M., Suffczynski, P., Lopes da Silva, F.H., 1999. Dynamics of the human alpha rhythm: evidence for non-linearity? Clin. Neurophysiol. 110, 1801-1813.

Tallon-Baudry, C., Bertrand, O., 1999. Oscillatory gamma activity in humans and its role in object representation. Trends Cogn. Sci. 3 (4), $151-162$.

Tallon-Baudry, C., Bertrand, O., Delpuech, C., Pernier, J., 1996. Stimulus specificity of phase-locked and non-phase-locked $40(\mathrm{~Hz}$ visual responses in human. J. Neurosci. 16 (13), 4240-4249.

Treue, S., Martinez-Trujillo, J.C., 1999. Feature-based attention influences motion processing gain in macaque visual cortex. Nature 399 (575), 579 .

Truccolo, W.A., Ding, M., Knuth, K.H., Nakamura, R., Bressler, S.L., 2002. Trial-to-trial variability of cortical evoked responses: implications for the analysis of functional connectivity. Clin. Neurophysiol. 113, $206-226$

Turrigiano, G.G., Nelson, S.B., 2004. Homeostatic plasticity in the developing nervous system. Nat. Rev., Neurosci. 5 (97), 107.

Varela, F.J., 1995. Resonant cell assemblies: a new approach to cognitive functions and neural synchrony. Biol. Res. 28, 81-95.

Wendling, F., Bellanger, J.J., Bartolomei, F., Chauvel, P., 2000. Relevance of nonlinear lumped-parameter models in the analysis of depth- EEG epileptic signals. Biol. Cybern. 83, 367-378. 